\title{
Identification and functional analysis of the L-ascorbate-specific enzyme II complex of the phosphotransferase system in Streptococcus mutans
}

\author{
Xinyu $\mathrm{Wu}^{1+}$, Jin $\mathrm{Hou}^{1+}$, Xiaodan Chen ${ }^{2}$, Xuan Chen $^{1}$ and Wanghong Zhao ${ }^{1 *}$
}

\begin{abstract}
Background: Streptococcus mutans is the primary etiological agent of human dental caries. It can metabolize a wide variety of carbohydrates and produce large amounts of organic acids that cause enamel demineralization. Phosphoenolpyruvate-dependent sugar phosphotransferase system (PTS) plays an important role in carbohydrates uptake of S. mutans. The $p t x A$ and $p t x B$ genes in S. mutans encode putative enzyme IIA and enzyme IIB of the L-ascorbate-specific PTS. The aim of this study was to analyze the function of these proteins and understand the transcriptional regulatory mechanism.
\end{abstract}

Results: $p t x A^{-}, p t \times B^{-}$, as well as $p t \times A^{-}, p t \times B^{-}$double-deletion mutants all had more extended lag phase and lower growth yield than wild-type strain UA159 when grown in the medium using L-ascorbate as the sole carbon source. Acid production and acid killing assays showed that the absence of the $p t x A$ and $p t x B$ genes resulted in a reduction in the capacity for acidogenesis, and all three mutant strains did not survive an acid shock. According to biofilm and extracellular polysaccharides (EPS) formation analysis, all the mutant strains formed much less prolific biofilms with small amounts of EPS than wild-type UA159 when using L-ascorbate as the sole carbon source. Moreover, PCR analysis and quantitative real-time PCR revealed that sgaT, ptxA, ptxB, SMU.273, SMU.274 and SMU.275 appear to be parts of the same operon. The transcription levels of these genes were all elevated in the presence of L-ascorbate, and the expression of ptxA gene decreased significantly once $p t \times B$ gene was knockout.

Conclusions: The ptxA and ptxB genes are involved in the growth, aciduricity, acidogenesis, and formation of biofilms and EPS of S. mutans when L-ascorbate is the sole carbon source. In addition, the expression of ptxA is regulated by ptxB. ptxA, ptxB, and the upstream gene sgaT, the downstream genes SMU.273, SMU.274 and SMU.275 appear to be parts of the same operon, and L-ascorbate is a potential inducer of the operon.

Keywords: Streptococcus mutans, Phosphotransferase system, L-ascorbate, Aciduricity, Acidogenesis, Biofilm formation, Extracellular polysaccharides

\footnotetext{
* Correspondence: zhaowh@smu.edu.cn

${ }^{\dagger}$ Equal contributors

${ }^{1}$ Department of Stomatology, Nanfang Hospital and College of Stomatology,

Southern Medical University, Guangzhou, Guangdong, China

Full list of author information is available at the end of the article
} 


\section{Background}

Streptococcus mutans is the primary etiological agent of human dental caries. It can metabolize a wide variety of carbohydrates that exist in the human oral cavity and produce large amounts of organic acids via the glycolytic pathway [1]. These metabolic byproducts cause a substantial drop in the $\mathrm{pH}$ of the oral cavity that in turn can result in the demineralization of enamel. The mechanisms of transport and metabolism of carbohydrates by S. mutans are therefore crucial to the onset and development of dental caries.

Although there are examples of carbohydrates that are internalized through ATP-binding cassette transporters (ABC transporters) [2], or other pathways [3-5], the dominant, high-affinity, high-capacity mechanism to transport and concomitantly phosphorylate carbohydrates in $S$. mutans is the phosphoenolpyruvate (PEP)-dependent sugar phosphotransferase system (PTS) [6]. More than 14 unique PTS permeases that transport a spectrum of carbohydrates including glucose [7, 8], sucrose [9], mannose [10], sorbitol [11], fructose [12], lactose [13], galactose $[14,15]$, maltose [16] and nigerose [17] are present in the reference strain $S$. mutans UA159. The PTS is usually composed of two general energy-coupling proteins that participate in the phosphorylation of all PTS substrates-the enzyme I (EI) and histidine-containing phosphocarrier protein $(\mathrm{HPr})$ and a series of substrate-specific permeases, known as enzyme II (EII) complexes, which are directly responsible for the transportation and phosphorylation of the substrates [6]. In most cases, the EII complexes are comprised of three functional domains, A, $\mathrm{B}$, and $\mathrm{C}$, but sometimes a fourth domain, $\mathrm{D}$, is required. The EIIA and EIIB domains are located in the cytoplasm and take part in the phosphorylation of the cognate substrates, while the EIIC and EIID domains act as the transmembrane channel and the sugar-binding site [18]. The PTS phosphorylates carbohydrates at the expense of PEP. During the transport process, the phosphoryl group on PEP is transferred to EI, then to a histidine residue on HPr, then to EIIA and EIIB, and finally to EIIC, forming a sugar-phosphate [18]. To date, a number of sugar-specific PTS of $S$. mutans have been further studied, such as glucose, sucrose, fructose, mannose, sorbitol, etc. Howerer, the study of L-ascorbate-specific PTS of S. mutans is little.

Previous studies have reported that some enteric bacteria can ferment and oxidize L-ascorbate under anaerobic conditions [19-21]. The metabolism of L-ascorbate has been described in detail in Escherichia coli [22-24], Lactobacillus [25] and Pneumobacillus [26, 27], but no study has formally shown that $S$. mutans can ferment this compound. In natural environment, the energy supply for growth and survival is often a limiting factor, organisms regularly encounter such energy-limited conditions, and they are forced to scavenge energy from all potential sources. L-ascorbate is abundant in many fruits and vegetables, so to study on the L-ascorbate-specific PTS that oral streptococci can use to obtain carbon sources is important.

Currently, six genes, ptxA, ptxB, sgaT, SMU.273, $S M U .274$ and SMU.275, analogous to the ula regulon used by $E$. coli to catabolize L-ascorbate under anaerobic conditions, have been identified in the S. mutans genome. These genes encode putative EIIA, EIIB, and EIIC of the L-ascorbate-specific PTS of S. mutans and three catabolic enzymes in the pentose phosphate pathway. Recently, the crystal structures of the PtxA (PDB: 3BJV) and PtxB (PDB: 3CZC) proteins have been analyzed [28]. Specific hydrophobic structures between these two proteins allow for efficient transfer of the phosphoryl group from PtxA to PtxB and then to the substrate.

In the present study, we knocked out the putative Lascorbate-specific EIIA gene ( $p t x A)$ and EIIB gene $(p t x B)$, individually and together, in $S$. mutans UA159, to explore their function. The results indicate that $p \operatorname{txA}$ and $p t x B$ are involved in growth, aciduricity, acidogenesis, and formation of biofilms and extracellular polysaccharides (EPS) when $S$. mutans is grown with L-ascorbate as the sole carbon source. Moreover, the expression of $p t x A$ is regulated by $p t x B$. $p t x A, p t x B$, and the adjacent genes sgaT, SMU.273, SMU.274 and SMU.275 are parts of the same operon, and L-ascorbate is a potential inducer of the operon.

\section{Methods}

\section{Bacterial strains, plasmids, and culture conditions}

The S. mutans strains and plasmids used in this study are listed in Table 1. S. mutans UA159 and its derivatives were routinely grown in brain-heart infusion (BHI) medium (Hopebio, Qingdao, Shandong, China) or tryptone-vitamin (TV) base medium [29] supplemented with $15 \mathrm{mM} \mathrm{L-}$ ascorbate (Sigma, St Louis, MO, USA) or glucose (Sigma, St Louis, MO, USA) as the sole carbon source, which were referred to as TVL medium and TVG medium, respectively. When needed, $1 \mathrm{mg} \mathrm{mL}^{-1}$ spectinomycin (Sigma, St Louis, MO, USA) was added to the medium. All bacterial cultures were incubated without agitation in an anaerobic atmosphere $\left(10 \% \mathrm{CO}_{2}\right.$, $10 \% \mathrm{H}_{2}, 80 \% \mathrm{~N}_{2}$ ) at $37{ }^{\circ} \mathrm{C}$, unless specified otherwise.

\section{Construction of $p t x A^{-}, p t x B^{-}$, and $p t x A^{-}, p t x B^{-}$double deletion mutants and complemented strains}

The procedure for generating the plasmid for construction of a $p t x A^{-}$strain was described previously [30]. Briefly, the 5' and 3' regions flanking the $p t x A$ gene were amplified from the genomic DNA of S. mutans UA159 by polymerase chain reaction (PCR) using the primers shown in Additional file 1: Table S1. Following proper restriction enzyme digestions, the flanking regions were 
Table 1 Bacterial strains and plasmids used in this study

\begin{tabular}{|c|c|c|}
\hline Strains or plasmids & Relevant characteristics & Source or reference \\
\hline \multicolumn{3}{|l|}{ Strains } \\
\hline S. mutans UA159 & Wild-type, serotype c & Ajdić et al., (2002) [4] \\
\hline S. mutans $p t x A^{-}$ & UA159 derivative, $\triangle p t x A^{-}$, Spe $^{r}$ & This study \\
\hline S. mutans $p t \times B^{-}$ & UA159 derivative, $\triangle p t x B^{-}$, Spe $^{r}$ & This study \\
\hline S. mutans ptxAB- & UA159 derivative, $\triangle p t \times A^{-}$and $\triangle p t \times B^{-}$, Spe ${ }^{r}$ & This study \\
\hline S. mutans CptxA $A^{-}$ & S. mutans ptxA $A^{-}$carrying pDL278:ptxA, Spe ${ }^{r}$ & This study \\
\hline S. mutans $C p t \times B^{-}$ & S. mutans $p t \times B^{-}$carrying $p D L 278: p t \times B$, Spe ${ }^{r}$ & This study \\
\hline S. mutans CptXAB- & S. mutans ptxAB- carrying pDL278:ptxAB, Spe ${ }^{r}$ & This study \\
\hline \multicolumn{3}{|l|}{ Plasmids } \\
\hline pFW5 & Commercial cloning vector, Spe ${ }^{r}$ & Podbielski A et al., (1996) [52] \\
\hline pDL278 & Shuttle vector, Spe ${ }^{r}$ & LeBlanc \& Lee (1991) [53] \\
\hline pDL278:ptxA & Shuttle vector carrying $p t x A, S p e^{r}$ & This study \\
\hline pDL278:ptxB & Shuttle vector carrying $p t \times B$, Spe ${ }^{r}$ & This study \\
\hline pDL278:ptxAB & Shuttle vector carrying $p t \times A$ and $p t \times B$, Spe ${ }^{r}$ & This study \\
\hline
\end{tabular}

Spe $e^{r}$ spectinomycin resistance

cloned into two multiple cloning sites of plasmid pFW5 to generate pFW5A. Subsequently, plasmid pFW5A was used to transform the wild-type strain UA159, which resulted in replacement of the $p t x A$ gene by a nonpolar spectinomycin resistance $\left(\mathrm{Spe}^{\mathrm{r}}\right)$ marker via allelic exchange. The transformation was carried out in BHI medium in the presence of $10 \%$ heat-inactivated horse serum and $100 \mathrm{nM}$ competence-stimulating peptide (CSP) [31]. Spectinomycin-resistant transformants were isolated, further confirmed by PCR and sequencing, and named $S$. mutans $p t x A^{-}$strain. A similar technique was used to construct a $p t x B^{-}$deletion mutant and a $p t x A^{-}, p t x B^{-}$double deletion mutant, named $S$. mutans $p t x B^{-}$strain and $S$. mutans ptxAB $B^{-}$strain, respectively. For complementation of mutants, the $p \operatorname{txA} p \operatorname{ptx} B$, and $p t x A-p t x B$ coding sequences, plus the $\mathrm{P}_{g t f B}$ promoter [32], were amplified by PCR, digested and cloned directly into shuttle vector pDL278 to generate pDL278:ptx $A$, pDL278:ptxB, pDL278:ptxAB, respectively. After sequence confirmation, the correct plasmids were used for transformation of $S$. mutans $p t x A^{-}$, $p t x B^{-}$, and $p t x A B^{-}$strains, generating complemented strains $S$. mutans $C p t x A^{-}$, $C p t x B^{-}$and $C p t x A B^{-}$, respectively.

\section{Bacterial growth rates}

To measure the growth rates of $S$. mutans when using $\mathrm{L}$-ascorbate or glucose as the sole carbon source, wildtype strain UA159 was grown in BHI medium overnight, and the optical density at $600 \mathrm{~nm}\left(\mathrm{OD}_{600}\right)$ of the cultures was adjusted to 1.0. The adjusted cultures were inoculated 1:100 into fresh TVL or TVG medium. Data for plotting growth curves were collected by measuring changes in
$\mathrm{OD}_{600}$ at $2 \mathrm{~h}$ intervals using a spectrophotometer over a total period of $48 \mathrm{~h}$. To compare the growth rates among UA159 and its derivatives, overnight cultures were diluted 1:100 into fresh TVL medium, and $\mathrm{OD}_{600}$ values were measured at $2 \mathrm{~h}$ intervals for a total of $72 \mathrm{~h}$.

\section{Acid production assay}

Overnight cultures of wild-type S. mutans UA159 and its derivatives in BHI medium were diluted 1:100 with fresh TVL medium or fresh BHI medium and then incubated at $37{ }^{\circ} \mathrm{C}$ in an anaerobic atmosphere for $24 \mathrm{~h}$ and $48 \mathrm{~h}$. The $\mathrm{pH}$ of the supernatant in the media was measured at the beginning, and after $24 \mathrm{~h}$ or $48 \mathrm{~h}$ of incubation. The acidogenesis ability was calculated as the difference in $\mathrm{pH}$ values measured at specific incubation times $(\Delta \mathrm{pH})$.

\section{Acid killing assay}

The ability of the mutants to tolerate acid stress was determined by acid killing assays, as described previously $[33,34]$. Briefly, S. mutans strains were grown in TVL medium until $\mathrm{OD}_{600} \approx 0.3$, harvested by centrifugation at $3800 \times \mathrm{g}$ at $4{ }^{\circ} \mathrm{C}$ for $10 \mathrm{~min}$, washed once with $0.1 \mathrm{M}$ glycine (Sigma, St Louis, MO, USA), pH 7.0, then the cell pellets were resuspended in fresh TVL medium that was adjusted to $\mathrm{pH} 5.0$ with $\mathrm{HCl}$ to undergo an adaptive acid tolerance response. Following an additional hour of incubation, cells were harvested, washed and subjected to acid killing by incubating the strains in $0.1 \mathrm{M}$ glycine, $\mathrm{pH} 2.8$, for $0,15,30$, and $45 \mathrm{~min}$. The surviving cells were appropriately diluted, plated on $\mathrm{BHI}$ agar, and incubated in an anaerobic atmosphere at $37^{\circ} \mathrm{C}$ for $48 \mathrm{~h}$. 


\section{Biofilm and EPS formation analysis}

To evaluate the biomass and structure of the biofilms with confocal laser scanning fluorescence microscopy, $S$. mutans UA159 and its derivatives were incubated in BHI medium overnight, and new cultures were inoculated by diluting them 1:100 into fresh TVL medium and dispensing $5 \mathrm{~mL}$ aliquots into 6-well plates (Corning, NY, USA) with coverslips in each well. After $120 \mathrm{~h}$ of $37{ }^{\circ} \mathrm{C}$ anaerobic incubation, the formed biofilms were washed gently twice with sterile PBS to remove unbound bacteria and stained with SYTO9 (Molecular Probes, Eugene, OR, USA) for $15 \mathrm{~min}$ at room temperature in a dark room. After SYTO9 removal, biofilms were incubated in calcofluor white (Sigma, St Louis, MO, USA) to stain the EPS under identical conditions. Then the biofilms were washed gently twice with sterile PBS again and examined with an Olympus Fluoview FV10i confocal microscope (Olympus, Tokyo, Japan). For the detection of SYTO9 (green), we used the $488 \mathrm{~nm}$ line of the argon laser. For calcofluor white (blue), we used the $351 \mathrm{~nm}$ line. At least five independent fields were collected at $100 \times$ magnification per experiment and three independent experiments were performed. Image J was used to calculate the area that the biofilms covered.

\section{PCR analysis and quantitative real-time PCR}

To characterize the mechanism regulating expression of the $p t x A$ and $p t x B$ genes, total RNA was extracted and purified. Briefly, an overnight culture of S. mutans UA159 was added to TVL medium or TVG medium and grown to late exponential phase. The cells were disrupted with liquid nitrogen and the RNA was extracted with RNAiso reagent (Takara, Otsu, Shiga, Japan) and treated with DNase I (Thermo Scientific, Utena, Lithuania). After confirming the absence of DNA by PCR, the conversion of RNA into cDNA was carried out using the PrimeScript RT Master Mix protocol (Takara, Otsu, Shiga, Japan). PCR was performed on cDNA templates with specific primers that span the sequences $S M U .268$ to sgaT, sgaT to $p t x B, p t x B$ to $p t x A, p t x A$ to $S M U .273, S M U .273$ to SMU.274, SMU.274 to SMU.275 and SMU.275 to SMU.277 (Additional file 1: Table S1), using DNA of $S$. mutans UA159 as a positive control $[35,36]$. To evaluate the expression of $p t x A, p t x B$ and their adjacent genes under the influence of $15 \mathrm{mM} \mathrm{L}$-ascorbate (with $15 \mathrm{mM}$ glucose used as control), quantitative real-time PCR (qRT-PCR) was performed with specific primers (Additional file 1: Table S1) using the SYBR Premix Ex Taq Kit protocol (Takara, Otsu, Shiga, Japan). The qRTPCR amplification with primers to the 16S rRNA gene was used as a reference for normalization. Non-template controls were included to confirm the absence of primerdimer formation. In addition, expression of $p t x A$ gene in wild-type UA159 and $p t x B^{-}$strain was also evaluated by qRT-PCR.

\section{Statistical analysis}

Quantitative data were analyzed using the Independentsamples $t$-test or One-way ANOVA test, and a $P$ value $<0.05$ indicated statistically significant differences.

\section{Results \\ Deletion of $p t x A$ or $p t x B$ causes major defects in bacterial growth rates}

In initial experiments, we tested the growth of the wildtype $S$. mutans UA159 under anaerobic conditions in TV base medium supplemented with various concentrations of L-ascorbate as the sole carbon source. We found that, to some extent, the growth yield increased with increase in the L-ascorbate concentration. However, higher concentrations retarded or even stopped growth. Consequently, considering the terminal yield of bacteria and the extent of the lag phase, we selected $15 \mathrm{mM}$ as the optimal concentration of L-ascorbate. When grown in the two different media, S. mutans reached a growth plateau in TVL medium at about $36 \mathrm{~h}$, and in TVG medium at $14 \mathrm{~h}$. The maximal culture density $\left(\mathrm{OD}_{600}\right)$ in TVL medium was found to be reduced by more than one third of that in TVG medium (Fig. 1a). These results indicated that L-ascorbate could act as a carbon source for $S$. mutans to survive under anaerobic conditions, but not as effectively as glucose. The slow induction in TVL may in part account for its longer lag period and lower terminal yield.

Deletions of the $p t x A$ or $p t x B$ genes impaired the ability of $S$. mutans to grow when using L-ascorbate as the sole carbohydrate (Fig. 1b). After $72 \mathrm{~h}$ of $37{ }^{\circ} \mathrm{C}$ anaerobic incubation, wild-type UA159 reached an $\mathrm{OD}_{600}$ of 0.8 and its lag phase was $12 \mathrm{~h}$. However, compared with wild-type UA159, the $p t x A^{-}$strain had an extended lag phase and decreased growth yield. The lag phase of $p t x A^{-}$strain was $22 \mathrm{~h}$ and its maximal culture density was only 0.6 approximately. In addition, the growth of $p t x B^{-}$and $p t x A B^{-}$strains was decreased even more substantially. In the $72 \mathrm{~h}$ of incubation, they could hardly grow. As expected, the presence of the recombinant plasmids pDL278:ptxA and pDL278:ptxAB restored the anaerobic growth of the $C p t x A^{-}$and $C p t x A B^{-}$ strains on L-ascorbate, although the growth rates and the $\mathrm{OD}_{600}$ were lower after $72 \mathrm{~h}$ of incubation when compared to those of the wild-type UA159. However, the $C p t x B^{-}$ strain could not be complemented by inclusion of the plasmid pDL278:ptxB.

$p t x A$ and $p t x B$ deletions resulted in reduced acidogenesis As seen in the results of the acid production assay (Fig. 2), the wild-type UA159 and all mutant derivatives 

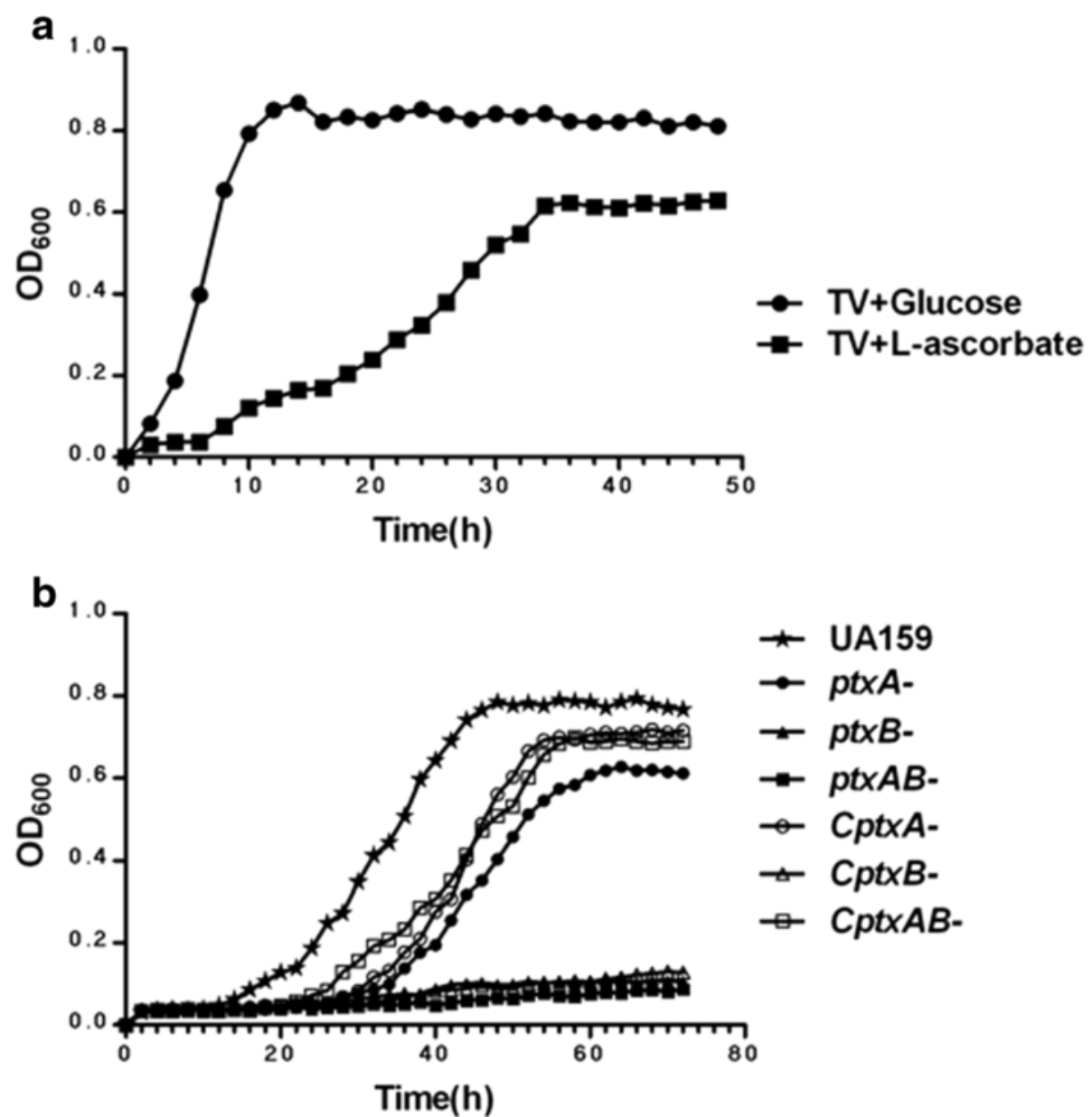

Fig. 1 Bacterial growth rates. a Growth of S. mutans UA159 incubated in TV medium supplemented with $15 \mathrm{mM}$ glucose $(\bullet)$ or L-ascorbate ( $\mathbf{\bullet})$

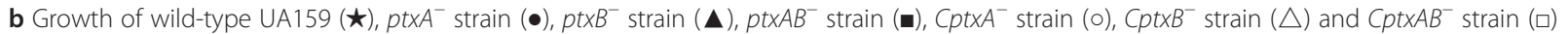
incubated in TV medium supplemented with $15 \mathrm{mM}$ L-ascorbate. Samples were all grown at $37^{\circ} \mathrm{C}$ for more than $48 \mathrm{~h}$ under anaerobic conditions and monitored every $2 \mathrm{~h}$ at $600 \mathrm{~nm}\left(\mathrm{OD}_{600}\right)$. The data presented here are the average of three independent experiments performed in triplicate

grew well and acidified the medium to about the same terminal $\mathrm{pH}$ in BHI medium after both $24 \mathrm{~h}$ and $48 \mathrm{~h}$ of incubation. The $\Delta \mathrm{pH}$ of the BHI medium was almost 0.95. However, in the case of the TVL medium, the terminal $\mathrm{pH}$ slightly decreased for all strains after incubation. The three mutants, and especially the $p t x B^{-}$and $p t x A B^{-}$strains, lowered the $\mathrm{pH}$ to a level significantly lower than that observed in wild-type UA159 culture after $24 \mathrm{~h}$ of incubation $(P<0.01)$. The $\triangle \mathrm{pH}$ of the TVL medium that $p t x A^{-}$strain, $p t x B^{-}$strain and $p t x A B^{-}$ strain grown in were $0.0567 \pm 0.0115,0.0233 \pm 0.0100$ and $0.0067 \pm 0.0057$, respectively. Furthermore, complemented strains recovered their acid production capacity, with the exception of the $C p t x B^{-}$strain. The reduced growth of the $\operatorname{Cptx} B^{-}$strain may account for its negligible $\mathrm{pH}$ change. Results that after $48 \mathrm{~h}$ of incubation were the same, except that all strains had produced more acid and the $\Delta \mathrm{pH}$ of the medium was greater than it was at $24 \mathrm{~h}$. $p t x A^{-}$and $p t x B^{-}$mutants did not survive an acid shock To determine the effects of $p t x A$ and $p t x B$ deletions on the ability to tolerate acid stress, the wild-type UA159 and single or double mutants were incubated in TVL medium with a $\mathrm{pH}$ of 5.0 for $1 \mathrm{~h}$ to induce an adaptive acid tolerance response and then were subjected to acid killing with a low-pH buffer ( $\mathrm{pH}$ 2.8). However, none of the mutant strains formed colonies on the assay plates following $15 \mathrm{~min}$ of low-pH incubation in triplicate tests, showing that the acid shock caused serious damage to the mutants.

Inactivation of the $p t x A$ and $p t x B$ genes affects biofilm and EPS formation in TVL medium

It could be seen from the results of confocal laser scanning fluorescence microscopy analysis, biofilms stained with the fluorescent dye SYTO9 appeared green (Fig. 3a) and EPS stained with calcofluor white appeared blue (Fig. 3b). The cover area of the biofilms formed by $S$. 


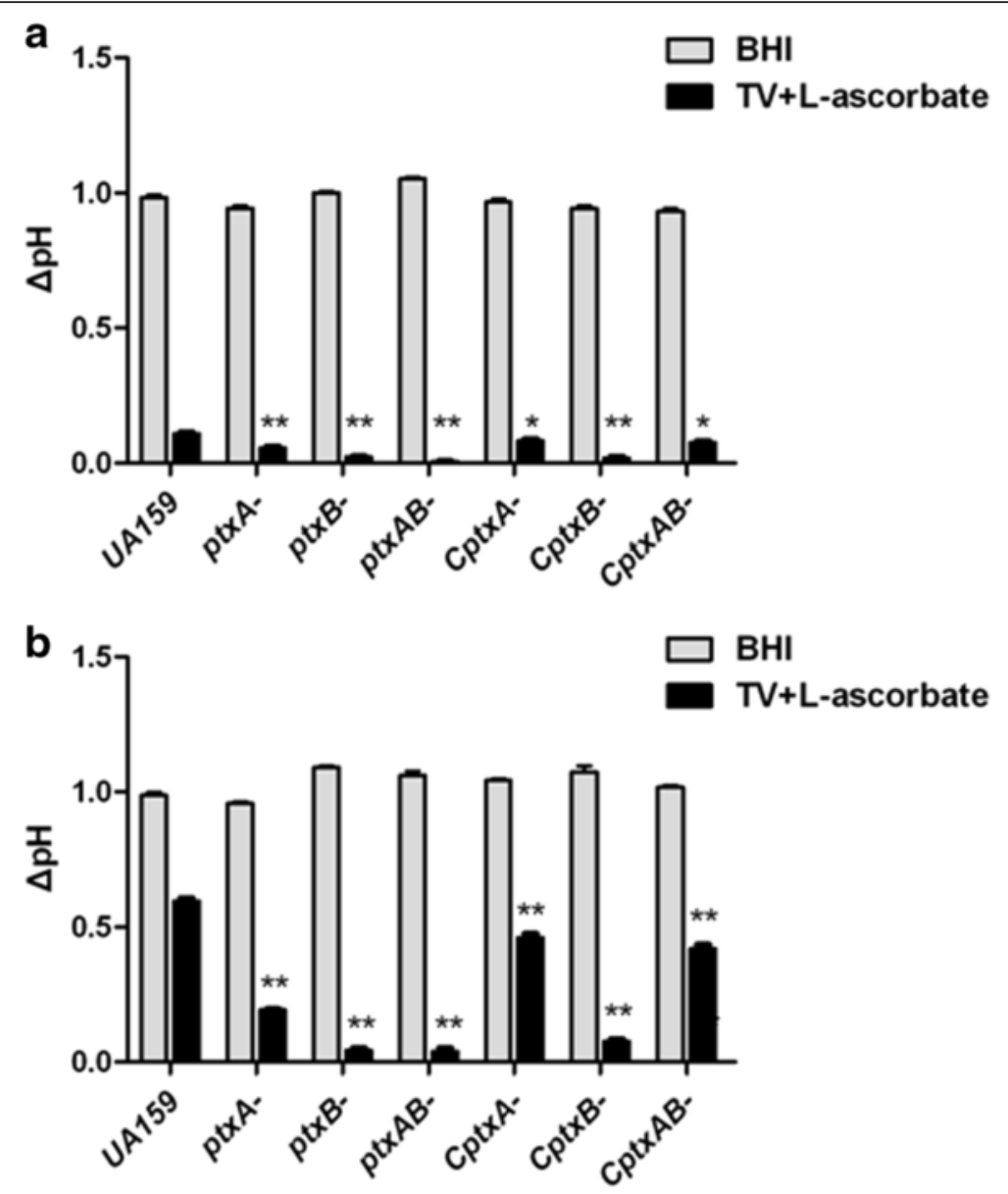

Fig. 2 Acid production assay. Wild-type UA159, $p t x A^{-}$strain, $p t x B^{-}$strain, $p t x A B^{-}$strain, $C p t x A^{-}$strain, $C p t x B^{-}$strain and $C p t x A B^{-}$strain were incubated in $\mathrm{BH}$ or TVL medium for $24 \mathrm{~h}(\mathbf{a})$ and $48 \mathrm{~h}(\mathbf{b})$ anaerobically. The $\mathrm{pH}$ measurements of the media were performed before and after the incubation, and are presented as $\Delta \mathrm{pH}$. A significant difference is indicated by ${ }^{*} P<0.05,{ }^{* *} P<0.01$ compared to UA159. The results presented here are the average of three independent experiments performed in triplicate

mutans UA159 and its derivatives was shown in Table 2. When using L-ascorbate as the sole carbon source, wildtype UA159 formed both small and large amorphous microcolonies and covered $65.93 \%$ of the surface. It created a thick and complex biofilms structure with a large amount of EPS. However, the biofilms and EPS $p t x A^{-}$strain formed were sparser and much thinner than UA159. It covered only $39.61 \%$ of the surface, but it still could form network structure. $p t x B^{-}$and $p t x A B^{-}$ strains formed much less prolific biofilms with only small amounts of EPS, in which cells were scattered on the surface as chains and the biofilms were too thin to form three-dimensional structure.

They covered only 24.24 and $18.57 \%$ of the surface respectively. Complementation in strains $\operatorname{Cptx} A^{-}$and $C p t x A B^{-}$restored biofilms and EPS formation to a level similar to that of wild-type UA159. However, $C p t x B^{-}$ strain could not restore the wild-type phenotype.
Transcriptional analysis of $p t x A, p t x B$ and their operon Transcriptional analysis using cDNA templates and primers that spanned the adjacent genes showed amplified bands in b, c, d, e and $\mathrm{f}$ regions (Fig. 4a) indicating that $p t x A, p t x B$, and the upstream gene sgaT, the downstream genes SMU.273, SMU.274 and SMU.275 are parts of the same operon. However, SMU.268 and SMU.277 are not parts of it. Quantitative real-time PCR (Fig. 4b) demonstrated that, compared with the gene expression in cells grown in medium containing glucose, the transcription level of these genes in cells grown in the presence of $15 \mathrm{mM} \mathrm{L}$-ascorbate were all elevated significantly $(P<0.01)$, further revealing that $p t x A, p t x B$, and the adjacent genes sgaT, SMU.273, SMU.274 and SMU.275 are parts of the same operon. In addition, the higher transcription levels of $p t x A$ and $p t x B$ genes in TV medium containing only L-ascorbate reinforced the finding that $S$. mutans could ferment $\mathrm{L}$-ascorbate to obtain energy under 


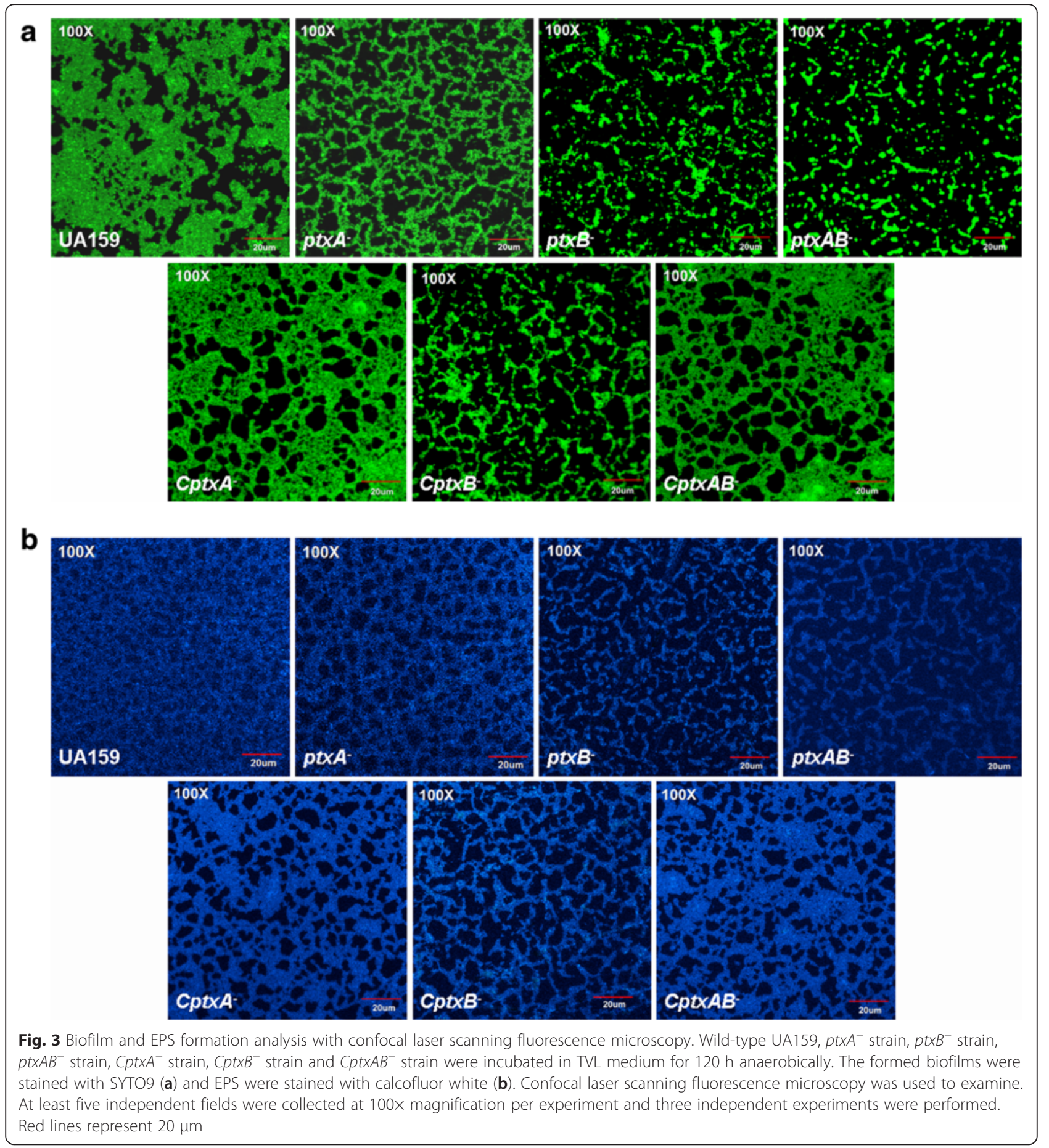

Table 2 Cover area of the biofilms formed by S. mutans UA159 and its derivatives

\begin{tabular}{llllllll}
\hline & UA159 & $p t x A^{-}$ & ptxB & ptxAB & CptxA $^{-}$ & CptxB $^{-}$ & CptxAB \\
\hline Cover area (\%) & 65.93 & 39.61 & 24.24 & 18.57 & 62.16 & 31.97 & 56.01 \\
\hline
\end{tabular}

anaerobic conditions, and suggested that the presence of L-ascorbate was required for up-regulation of transcription of $p t x A$ and $p t x B$. However, once $p t x B$ gene was knockout, the expression of $p t x A$ gene decreased significantly $(P<0.01)$ compared with wild-type UA159 (Fig. 4c). This result could well explain the finding in bacterial growth rates that why the wild-type phenotype could not be restored in the $C p t x B^{-}$strain. 


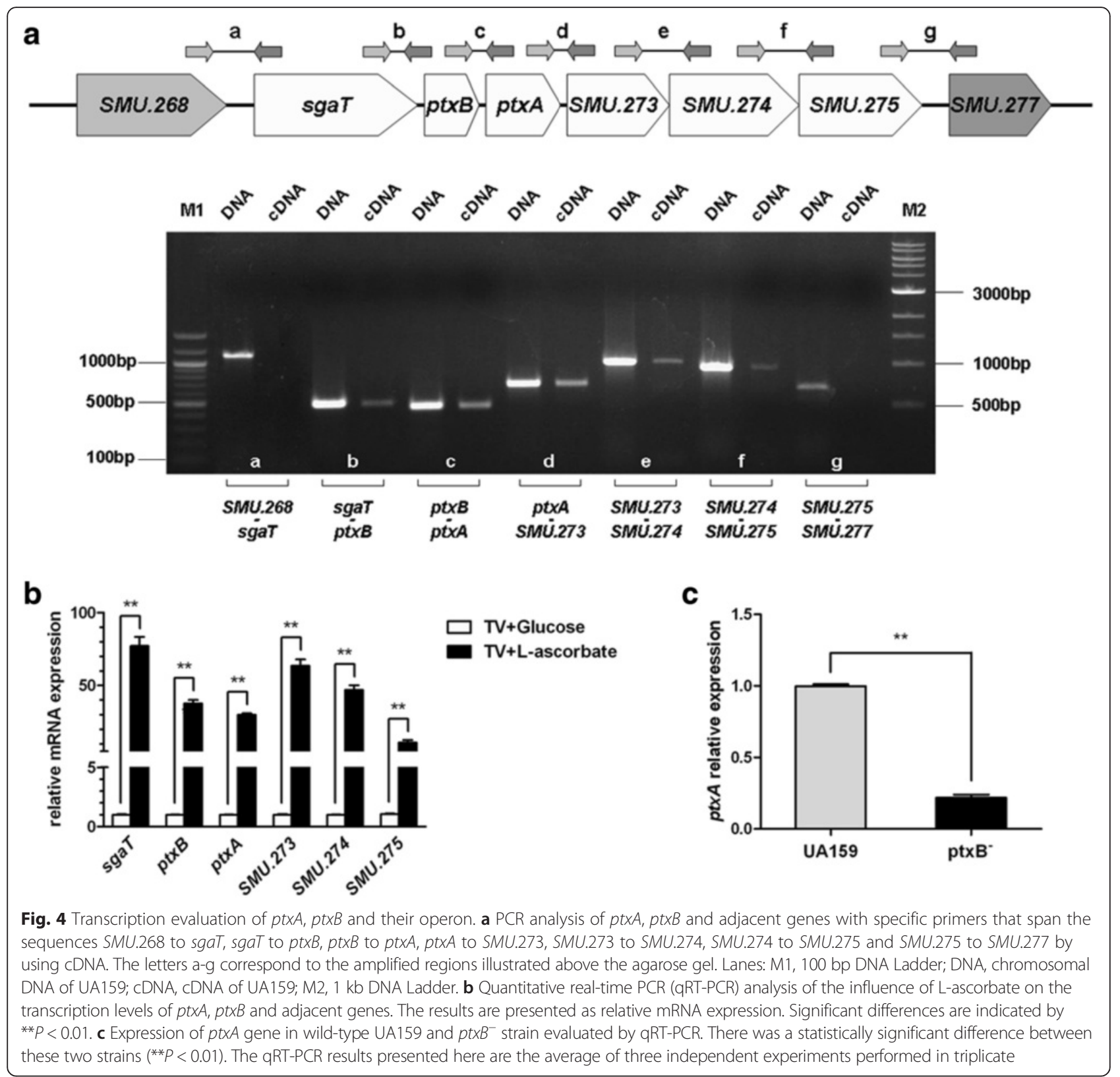

\section{Discussion}

Since the first discovery of PTS in E. coli [37], special efforts have been made to study the characteristics and functions of various PTS proteins in both gram-negative and gram-positive microorganisms, including S. mutans, the most common pathogen in dental caries. The presence of PTS involved in high-efficiency transport and phosphorylation of numerous carbohydrates largely accounts for the high cariogenicity of $S$. mutans. Apart from the two general proteins, EI and HPr, many genes coding for different carbohydrate-specific EII complexes of the PTS have been isolated and identified, such as the scrA gene for sucrose [38], the $m t l A$ gene for mannitol
[10], the lacFE genes for lactose [39], the manLMN genes for mannose [40], and others. In the present study, two genes, $p t x A$ and $p t x B$, that were identified and presumed to be involved in anaerobic utilization of $\mathrm{L}$ ascorbate, were analyzed.

Similar to $E$. coli and some other enteric bacteria, $S$. mutans could grow in defined medium supplemented with L-ascorbate as the sole energy. This provided evidence that $S$. mutans can obtain energy by fermenting this compound in an anaerobic atmosphere. However, at high concentrations, L-ascorbate failed to support the growth of $S$. mutans. This may be the result of an alteration of the internal redox state of the cells [23]. 
$\mathrm{L}$-ascorbate can trigger the Fenton reaction in the presence of redox-active iron and oxygen, which yields ROS from hydrogen peroxide and leads to oxidative stress $[41,42]$. Moreover, although L-ascorbate is an effective antioxidant, $\mathrm{H}_{2} \mathrm{O}_{2}$ will be released from its oxidation and can cause damage to cells [43]. The deletion of the $p t x A$ and $p t x B$ genes seriously affected the growth of $S$. mutans when using L-ascorbate as the sole carbon source, which indicated that the $p t x A$ and $p t x B$ gene products are indeed involved in the anaerobic dissimilation of L-ascorbate. However, the deletion of $p t x B$ caused a more severe impact on cell growth than deletion of $p t x A$, and the wild-type phenotype could not be restored in the $C p t x B^{-}$strain. This suggests that the $p t x B$ gene, or its product, seems to be more important in this metabolic process. Previous study has found that the interaction of PtxA and PtxB proteins of $S$. mutans is weak [28], which increased the complexity of the phosphoryl transfer mechanism of L-ascorbate-specific PTS of S. mutans. Based on our experimental results, we have reasons to believe that ptxB plays a more essential role in the phosphoryl transfer. Another reasonable interpretation is that deletion of the $p t x B$ gene caused a polar effect on the downstream $p t x A$ gene, in which case complementation by $p t x B$ could not restore the phenotype.

S. mutans has the ability to produce organic acids and cause enamel demineralization, so acidogenic capacity plays a crucial role in the occurrence of caries. The acid production assay indicated that an absence of $p t x A$ and $p t x B$ genes leads to lower glycolytic activities. This weakened capacity for acidogenesis is likely attributed to the reduced ability to grow, as reflected by the reduced growth rates and culture densities. Additionally, the $\mathrm{pH}$ of the medium can also affect the ability of glycolytic activity to lower the external $\mathrm{pH}$. At lower $\mathrm{pH}$, the cellular metabolism and energy levels are lower due to repression of amino acid synthesis genes. In addition, both glycolytic activity and amino acid biosynthesis require $\mathrm{NAD}^{+}$as a cofactor $[44,45]$. Since the total intracellular $\mathrm{NAD}^{+}$pool is limited, competition between these two processes for $\mathrm{NAD}^{+}$should slow down both pathways [46].

It is well known that $S$. mutans possesses an acidtolerance response and the ability to tolerate acid stress will elevate after initial incubation in low $\mathrm{pH}$ medium $[47,48]$. However, according to the results from the acid killing assay, none of the three mutants could survive in a buffer with a $\mathrm{pH}$ of 2.8 , even after a $1 \mathrm{~h}$ acid adaptation. This showed that absence of the $p t x A$ and $p t x B$ genes resulted in the loss of the ability to tolerate acid stress when using L-ascorbate as the sole carbon source. The possible reason may be that L-ascorbate is not the most optimistic carbon source for $S$. mutans to ferment. The strains, on one hand, were under nutritional stress, and on the other hand, were under acidic stress. Oral streptococci often encounter acid stress conditions in the oral cavity. Therefore, the ability to survive acidic conditions may play a crucial role in the growth of these bacteria. Proton extrusion by the membrane-associated F-ATPase is the primary mechanism employed by $S$. mutans to maintain intracellular $\mathrm{pH}$ homeostasis [49], and mutations in some genes can have an impact on the conformation of, and functional capacity to extrude protons by, the F-ATPase enzyme [33]. However, the mechanism by which the absence of the $p t x A$ and $p t x B$ genes hinders the acid tolerance response remains to be discovered.

Biofilm formation is an important pathogenic trait that allows bacteria to attach to and colonize the tooth surface. Glucosyltransferases (GTFs) and glucanbinding proteins (GBPs) always play important roles in the process. However, when using L-ascorbate as the sole carbon source, UA159 still could form prolific biofilms and EPS, with no sucrose or glucose exist. We speculated that the limited nutritional stress was responsible for the prolific biofilm and EPS formation [46]. In TVL medium, L-ascorbate was the only energy source to support growth, and this may have automatically triggered biofilm formation because $S$. mutans is adapted to biofilm formation as its primary life style. What's more, the difference of biofilm and EPS formation capacity among wild-type strain, mutant strains and complemented strains is likely attributed to the difference of bacterial growth rates.

The carbohydrate-specific PTS catalyze the concomitant transport and phosphorylation of their sugar substrates [6]. So far, 45 homologous L-ascorbate phosphotransferase transport systems from a wide variety of bacteria have been identified [50]. These systems fall into five structural types, and in S. mutans, EIIA, EIIB, and EIIC are encoded by distinct genes. The $p t x A$ and $p t x B$ genes of $S$. mutans encode the putative EIIA and EIIB of the L-ascorbatespecific PTS, and sgaT encodes the putative EIIC. What's more, the downstream gene SMU.273 encodes 3-keto-Lgulonate-6-phosphate decarboxylase, SMU.274 encodes L-xylulose 5-phosphate 3-epimerase, and SMU.275 encodes L-ribulose-5-phosphate 4-epimerase. They also play essential roles in L-ascorbate metabolism. Based on the PCR analysis using cDNA as template, sgaT, $p t x B, p t x A$, SMU.273, SMU.274 and SMU.275 appear be parts of the same operon. This is similar to the ulaA-F operon in $E$. coli [51] that encodes the three components of the Lascorbate phosphotransferase transport system (ulaABC), as well as three catabolic enzymes (ulaDEF). The ulaA, $u l a B$, and $u l a C$ gene products are involved in the uptake and phosphorylation of L-ascorbate, and the ulaD, ulaE, and $u l a F$ gene products are involved in the subsequent metabolism by the pentose phosphate pathway [23, 24]. Based 
on the result that sgaT, $p t x A, p t x B, S M U .273, S M U .274$ and $S M U .275$ could be all up-regulated significantly in the presence of L-ascorbate, it was concluded in this study that $\mathrm{L}$-ascorbate is a potential inducer of the operon.

\section{Conclusion}

This work indicates that $p t x A$ and $p t x B$ genes, encode putative enzyme IIA and enzyme IIB of the L-ascorbatespecific PTS in S. mutans, influence the physiology and virulence of $S$. mutans, including the growth rate, the capacity of aciduricity, acidogenesis, and formation of biofilm and EPS when using L-ascorbate as the sole carbon source. In addition, the expression of $p t x A$ is regulated by $p t x B$. $p t x A, p t x B$, and the adjacent genes sgaT, SMU.273, SMU.274 and SMU.275 are parts of the same operon, and L-ascorbate is a potential inducer of the operon. Functional analysis of genes in PTS of the primary cariogenic etiological agent is crucial to the prevention and treatment of dental caries. Current efforts are being directed toward gaining a better understanding of how these genes are regulated, and to reveal further insights into their roles in metabolic pathways.

\section{Availability of data and materials}

The data supporting the conclusions of this article are included within the article and additional file.

\section{Additional file}

Additional file 1: Table S1. Primers used in this study. (DOC $62 \mathrm{~kb}$ )

\section{Abbreviations}

ABC transporters: ATP-binding cassette transporters; BHI: brain-heart infusion; CSP: competence-stimulating peptide; El: enzyme I; Ell: enzyme II; EPS: extracellular polysaccharides; GBPs: glucan-binding proteins: GTFs: glucosyltransferases; HPr: histidine-containing phosphocarrier protein; OD: optical density; PCR: polymerase chain reaction; PEP: phosphoenolpyruvate; PTS: phosphotransferase system; qRT-PCR: quantitative real-time PCR; Sper: spectinomycin resistance; TV: tryptone-vitamin; TVG: tryptone-vitamin supplemented with glucose; TVL: tryptone-vitamin supplemented with L-ascorbate.

\section{Competing interests}

The authors declare that they have no competing interests.

\section{Authors' contributions}

WZ and $\mathrm{JH}$ contributed to the design and coordination of the study. XW, Xiaodan Chen and Xuan Chen carried out the laboratory experiments. XW and Xuan Chen contributed to the analysis of results. XW and Xiaodan Chen drafted the manuscript. All authors read and approved the final manuscript.

\section{Acknowledgments}

We thank Prof. Qing Yu (School of Stomatology, Fourth Military Medical University, China) for providing pFW5 plasmid, Prof. Zezhang T. Wen (Center of Excellence in Oral and Craniofacial Biology, School of Dentistry, Louisiana State University Health Sciences Center, USA) for providing pDL278 plasmid, and Prof. Jiman He (Department of Gastroenterology, Nanfang Hospital, Southern Medical University, China) for valuable assistance with anaerobic bacteria incubation. This work was supported by National Natural Science Foundation of China (81050035), Guangdong University Development
Foundation and the President Foundation of Nanfang Hospital, Southern Medical University (2013B003) to Wanghong. Zhao.

\section{Author details}

${ }^{1}$ Department of Stomatology, Nanfang Hospital and College of Stomatology, Southern Medical University, Guangzhou, Guangdong, China. ${ }^{2}$ Department of Stomatology, the Second Affiliated Hospital of Shantou University, Shantou, Guangdong, China.

Received: 22 September 2015 Accepted: 7 March 2016

Published online: 22 March 2016

\section{References}

1. Drucker DB, Melville TH. Fermentation end-products of cariogenic and non-cariogenic streptococci. Arch Oral Biol. 1968;13:565-70.

2. Russell RR, Aduse-Opoku J, Sutcliffe IC, Tao L, Ferretti JJ. A binding protein-dependent transport system in Streptococcus mutans responsible for multiple sugar metabolism. J Biol Chem. 1992;267:4631-7.

3. Ajdić D, Sutcliffe IC, Russell RR, Ferretti JJ. Organization and nucleotide sequence of the Streptococcus mutans galactose operon. Gene. 1996;180:137-44.

4. Ajdić D, McShan WM, McLaughlin RE, et al. Genome sequence of Streptococcus mutans UA159, a cariogenic dental pathogen. Proc Natl Acad Sci U S A. 2002:99:14434-9.

5. Ajdić D, Pham VT. Global transcriptional analysis of Streptococcus mutans sugar transporters using microarrays. J Bacteriol. 2007;189:5049-59.

6. Postma PW, Lengeler JW, Jacobson GR. Phosphoenolpyruvate:carbohydrate phosphotransferase systems of bacteria. Microbiol Rev. 1993;57:543-94.

7. Schachtele CF, Mayo JA. Phosphoenolpyruvate-dependent glucose transport in oral streptococci. J Dent Res. 1973:52:1209-15.

8. Cvitkovitch DG, Boyd DA, Thevenot T, Hamilton IR. Glucose transport by a mutant of Streptococcus mutans unable to accumulate sugars via the phosphoenolpyruvate phosphotransferase system. J Bacteriol. 1995;177:2251-8.

9. Zeng L, Burne RA. Comprehensive mutational analysis of sucrosemetabolizing pathways in Streptococcus mutans reveals novel roles for the sucrose phosphotransferase system permease. J Bacteriol. 2013;195:833-43.

10. Honeyman AL, Curtiss R. The mannitol-specific enzyme II (mt|A) gene and the mtIR gene of the PTS of Streptococcus mutans. Microbiology. 2000;146:1565-72

11. Boyd DA, Thevenot T, Gumbmann M, Honeyman AL, Hamilton IR. Identification of the operon for the sorbitol (Glucitol) Phosphoenolpyruvate: Sugar phosphotransferase system in Streptococcus mutans. Infect Immun. 2000;68:925-30

12. Bourassa $\mathrm{S}$, Gauthier L, Giguère R, Vadeboncoeur C. A IIIman protein is involved in the transport of glucose, mannose and fructose by oral streptococci. Oral Microbiol Immunol. 1990;5:288-97.

13. Honeyman AL, Curtiss R. Isolation, characterization and nucleotide sequence of the Streptococcus mutans lactose-specific enzyme II (lacE) gene of the PTS and the phospho-beta-galactosidase (lacG) gene. J Gen Microbiol. 1993;139:2685-94.

14. Zeng L, Das S, Burne RA. Utilization of lactose and galactose by Streptococcus mutans: transport, toxicity, and carbon catabolite repression. J Bacteriol. 2010;192:2434-44.

15. Zeng $L$, Xue P, Stanhope MJ, Burne RA. A galactose-specific sugar: phosphotransferase permease is prevalent in the non-core genome of Streptococcus mutans. Mol Oral Microbiol. 2013;28:292-301.

16. Webb AJ, Homer KA, Hosie AH. A phosphoenolpyruvate-dependent phosphotransferase system is the principal maltose transporter in Streptococcus mutans. J Bacteriol. 2007:189:3322-7.

17. Ajdić D, Chen Z. A novel phosphotransferase system of Streptococcus mutans is responsible for transport of carbohydrates with a-1, 3 linkage. Mol Oral Microbiol. 2013;28:114-28.

18. Saier Jr MH, Reizer J. Proposed uniform nomenclature for the proteins and protein domains of the bacterial phosphoenolpyruvate: sugar phosphotransferase system. J Bacteriol. 1992;174:1433-8.

19. Esselen WB, Fuller JE. The oxidation of ascorbic acid as influenced by intestinal bacteria. J Bacteriol. 1939;37:501-21.

20. Young RM, James LH. Action of intestinal microorganisms on ascorbic acid. J Bacteriol. 1942:44:75-84. 
21. Volk WA, Larsen JL. beta-Keto-L-gulonic acid as an intermediate in the bacterial metabolism of ascorbic acid. J Biol Chem. 1962;237:2454-7.

22. Campos E, Aguilar J, Baldoma L, Badia J. The gene yjfQ encodes the repressor of the yjfR-X regulon (Ula), which is involved in L-ascorbate metabolism in Escherichia coli. J Bacteriol. 2002;184:6065-8.

23. Yew WS, Gerlt JA. Utilization of L-ascorbate by Escherichia coli K-12: assignments of functions to products of the yjf-sga and yia-sgb operons. J Bacteriol. 2002;184:302-6.

24. Zhang Z, Aboulwafa M, Smith MH, Saier Jr MH. The ascorbate transporter of Escherichia coli. J Bacteriol. 2003;185:2243-50.

25. Linares D, Michaud P, Delort AM, Traïkia M, Warrand J. Catabolism of L-ascorbate by Lactobacillus rhamnosus GG. J Agric Food Chem. 2011;59:4140-7

26. Campos E, de la Riva L, Garces F, et al. The yiaKLX1X2PQRS and ulaABCDEFG gene systems are required for the aerobic utilization of L-ascorbate in Klebsiella pneumoniae strain 13882 with L-ascorbate-6phosphate as the inducer. J Bacteriol. 2008;190:6615-24.

27. Campos E, Aguilera L, Giménez R, Aguilar J, Baldoma L, Badia J. Role of YiaX2 in I-ascorbate transport in Klebsiella pneumoniae 13882. Can J Microbiol. 2009;55:1319-22.

28. Lei J, Li LF, Su XD. Crystal structures of phosphotransferase system enzymes PtxB (IIB(Asc)) and PtxA (IIA(Asc)) from Streptococcus mutans. J Mol Biol. 2009;386:465-75

29. Burne RA, Wen ZT, Chen YY, Penders JE. Regulation of expression of the fructan hydrolase gene of Streptococcus mutans GS-5 by induction and carbon catabolite repression. J Bacteriol. 1999;181:2863-71.

30. Lau PC, Sung CK, Lee JH, Morrison DA, Cvitkovitch DG. PCR ligation mutagenesis in transformable streptococci: application and efficiency. J Microbiol Methods. 2002:49:193-205.

31. Petersen FC, Scheie AA. Natural transformation of oral streptococci. Methods Mol Biol. 2010;666:167-80

32. Biswas I, Jha JK, Fromm N. Shuttle expression plasmids for genetic studies in Streptococcus mutans. Microbiology. 2008;154:2275-82.

33. Wen ZT, Burne RA. LuxS-mediated signaling in Streptococcus mutans is involved in regulation of acid and oxidative stress tolerance and biofilm formation. J Bacteriol. 2004;186:2682-91.

34. Bitoun JP, Liao S, Xie GG, Beatty WL, Wen ZT. Deficiency of BrpB causes major defects in cell division, stress responses and biofilm formation by Streptococcus mutans. Microbiology. 2014;160:67-78.

35. Ardin AC, Fujita K, Nagayama K, et al. Identification and functional analysis of an ammonium transporter in Streptococcus mutans. PLoS One. 2014;9: e107569.

36. Faustoferri RC, Hubbard CJ, Santiago B, Buckley AA, Seifert TB, Quivey Jr RG. Regulation of fatty acid biosynthesis by the global regulator CcpA and the local regulator FabT in Streptococcus mutans. Mol Oral Microbiol. 2015;30: 128-46.

37. Kundig W, Ghosh S, Roseman S. Phosphate bound to histidine in a protein as an intermediate in a novel phospho-transferase system. Proc Natl Acad Sci U S A. 1964:52:1067-74

38. Sato Y, Poy F, Jacobson GR, Kuramitsu HK. Characterization and sequence analysis of the scrA gene encoding enzyme II Scr of the Streptococcus mutans phosphoenolpyruvatedependent sucrose phosphotransferase system. J Bacteriol. 1989;171:263-71.

39. Rosey EL, Stewart GC. Nucleotide and deduced amino acid sequences of the lacR, lacABCD, and lacFE genes encoding the repressor, tagatose 6phosphate gene cluster, and sugarspecific phosphotransferase system components of the lactose operon of Streptococcus mutans. J Bacteriol. 1992;174:6159-70

40. Sato Y, Okamoto-Shibayama K, Azuma T. Glucose-PTS involvement in maltose metabolism by Streptococcus mutans. Bull Tokyo Dent Coll. 2015; 56:93-103.

41. Pericone CD, Park S, Imlay JA, Weiser JN. Factors contributing to hydrogen peroxide resistance in Streptococcus pneumoniae include pyruvate oxidase (SpxB) and avoidance of the toxic effects of the fenton reaction. J Bacteriol. 2003;185:6815-25.

42. Winterbourn CC. Toxicity of iron and hydrogen peroxide: the Fenton reaction. Toxicol Lett. 1995;82-83:969-74.

43. Richter HE, Loewen PC. Induction of catalase in Escherichia coli by ascorbic acid involves hydrogen peroxide. Biochem Biophys Res Commun. 1981;100: 1039-46.

44. Garvie El. Bacterial lactate dehydrogenases. Microbiol Rev. 1980;44:106-39.
45. Cvitkovitch DG, Gutierrez JA, Bleiweis AS. Role of the citrate pathway in glutamate biosynthesis by Streptococcus mutans. J Bacteriol. 1997;179:650-5.

46. Król JE, Biswas S, King C, Biswas I. SMU.746-SMU.747, a putative membrane permease complex, is involved in aciduricity, acidogenesis, and biofilm formation in Streptococcus mutans. J Bacteriol. 2014;196:129-39.

47. Assev S, Rolla G. Further studies on the growth inhibition of Streptococcus mutans OMZ 176 by xylitol. Acta Path Microbiol Immunol Scand SectB. 1986;94:97-102.

48. Davies D. Understanding biofilm resistance to antibacterial agents. Nat Rev Drug Discov. 2003;2:114-22.

49. Oxygen metabolism, oxidative stress and acid-base physiology of dental plaque biofilms. J Ind Microbiol. 1995;15:198-207.

50. Hvorup R, Chang AB, Saier Jr MH. Bioinformatic analyses of the bacterial Lascorbate phosphotransferase system permease family. J Mol Microbiol Biotechnol. 2003;6:191-205.

51. Campos E, Baldoma L, Aguilar J, Badia J. Regulation of expression of the divergent ulaG and ulaABCDEF operons involved in LaAscorbate dissimilation in Escherichia coli. J Bacteriol. 2004;186:1720-8.

52. Podbielski A, Spellerberg B, Woischnik M, Pohl B, Lütticken R. Novel series of plasmid vectors for gene inactivation and expression analysis in group A streptococci (GAS). Gene. 1996;177:137-147.

53. Leblanc D J, Lee L N, Inamine J M. Cloning and nucleotide base sequence analysis of a spectinomycin adenyltransferase $\operatorname{AAD}(9)$ determinant from Enterococcus faecalis. Antimicrob Agents Chemother. 1991;35:1804-1810.

\section{Submit your next manuscript to BioMed Central and we will help you at every step:}

- We accept pre-submission inquiries

- Our selector tool helps you to find the most relevant journal

- We provide round the clock customer support

- Convenient online submission

- Thorough peer review

- Inclusion in PubMed and all major indexing services

- Maximum visibility for your research

Submit your manuscript at www.biomedcentral.com/submit
) Biomed Central 